Silvestru Sever Dragomir (Melbourne and Johannesburg)

\title{
APPROXIMATING THE VOLUME INTEGRAL BY A SURFACE INTEGRAL VIA THE DIVERGENCE THEOREM
}

Abstract. By utilising the divergence theorem for $n$-dimensional integrals, we provide some error estimates for approximating the integral on a body $B$, a bounded closed subset of $\mathbb{R}^{n}(n \geq 2)$ with smooth (or piecewise smooth) boundary $\partial B$, by an integral on the surface $\partial B$ and some other simple terms. Some examples in the 3 -dimensional case are also given.

1. Introduction. Consider a closed, bounded and convex subset $D$ of $\mathbb{R}^{2}$. Denote by

$$
A_{D}:=\iint_{D} d x d y
$$

the area of $D$ and by $\left(\overline{x_{D}}, \overline{y_{D}}\right)$ the centre of mass of $D$, where

$$
\overline{x_{D}}:=\frac{1}{A_{D}} \iint_{D} x d x d y, \quad \overline{y_{D}}:=\frac{1}{A_{D}} \iint_{D} y d x d y .
$$

Let $f=f(x, y)$ be a function of two variables. We assume that the partial derivatives $\frac{\partial f}{\partial x}, \frac{\partial f}{\partial y}$ satisfy the Lipschitz type conditions at a point $(u, v) \in D$ :

$$
\left|\frac{\partial f}{\partial x}(x, y)-\frac{\partial f}{\partial x}(u, v)\right| \leq L_{1}|x-u|+K_{1}|y-v|
$$

and

$$
\left|\frac{\partial f}{\partial y}(x, y)-\frac{\partial f}{\partial y}(u, v)\right| \leq L_{2}|x-u|+K_{2}|y-v|
$$

for any $(x, y) \in D$, where $L_{1}, K_{1}, L_{2}$ and $K_{2}$ are given positive constants.

2020 Mathematics Subject Classification: Primary 26D15.

Key words and phrases: volume integral, surface integral, divergence theorem, integral inequalities.

Received 17 July 2019; revised 8 October 2019.

Published online 10 June 2020. 
In the recent paper [7] we established the following result on approximating the double integral by a contour integral:

TheOREM 1. Let $\partial D$ be a simple, closed counterclockwise oriented curve bounding a region $D$ and let $f$ be defined on an open set containing $D$ and having continuous partial derivatives on $D$. Assume that $(u, v) \in D$ and $\frac{\partial f}{\partial x}, \frac{\partial f}{\partial y}$ satisfy the Lipschitz type conditions $\sqrt{1.1}$ and $(1.2)$. Then for any $\alpha, \beta \in \mathbb{C}$ we have

$$
\begin{aligned}
& \mid \frac{1}{A_{D}} \iint_{D} f(x, y) d x d y \\
& \quad-\frac{1}{2 A_{D}} \oint_{\partial D}[(\beta-y) f(x, y) d x+(x-\alpha) f(x, y) d y] \\
& \quad-\frac{1}{2} \frac{\partial f}{\partial x}(u, v)\left(\alpha-\overline{x_{D}}\right)-\frac{1}{2} \frac{\partial f}{\partial y}(u, v)\left(\beta-\overline{y_{D}}\right) \mid \\
& \quad \leq \frac{L_{1}}{2 A_{D}} \iint_{D}|\alpha-x||x-u| d x d y+\frac{K_{1}}{2 A_{D}} \iint_{D}|\alpha-x||y-v| d x d y \\
& \quad+\frac{L_{2}}{2 A_{D}} \iint_{D}|\beta-y||x-u| d x d y+\frac{K_{2}}{2 A_{D}} \iint_{D}|\beta-y||y-v| d x d y .
\end{aligned}
$$

In particular,

$$
\begin{aligned}
& \mid \frac{1}{A_{D}} \iint_{D} f(x, y) d x d y \\
& -\frac{1}{2 A_{D}} \oint_{\partial D}\left[\left(\overline{y_{D}}-y\right) f(x, y) d x+\left(x-\overline{x_{D}}\right) f(x, y) d y\right] \mid \\
& \leq \frac{L_{1}}{2 A_{D}} \iint_{D}\left|\overline{x_{D}}-x\right||x-u| d x d y+\frac{K_{1}}{2 A_{D}} \iint_{D}\left|\overline{x_{D}}-x\right||y-v| d x d y \\
& +\frac{L_{2}}{2 A_{D}} \iint_{D}\left|\overline{y_{D}}-y\right||x-u| d x d y+\frac{K_{2}}{2 A_{D}} \iint_{D}\left|\overline{y_{D}}-y\right||y-v| d x d y
\end{aligned}
$$

and

$$
\begin{aligned}
& \mid \frac{1}{A_{D}} \iint_{D} f(x, y) d x d y \\
& -\frac{1}{2} \frac{\partial f}{\partial x}(u, v)\left(x_{f, \partial D}-\overline{x_{D}}\right)-\frac{1}{2} \frac{\partial f}{\partial y}(u, v)\left(y_{f, \partial D}-\overline{y_{D}}\right) \mid \\
& \leq \frac{L_{1}}{2 A_{D}} \iint_{D}\left|x_{f, \partial D}-x\right||x-u| d x d y+\frac{K_{1}}{2 A_{D}} \iint_{D}\left|x_{f, \partial D}-x\right||y-v| d x d y \\
& +\frac{L_{2}}{2 A_{D}} \iint_{D}\left|y_{f, \partial D}-y\right||x-u| d x d y+\frac{K_{2}}{2 A_{D}} \iint_{D}\left|y_{f, \partial D}-y\right||y-v| d x d y,
\end{aligned}
$$


where

$$
x_{f, \partial D}:=\frac{\oint_{\partial D} x f(x, y) d y}{\oint_{\partial D} f(x, y) d y} \quad \text { and } \quad y_{f, \partial D}:=\frac{\oint_{\partial D} y f(x, y) d x}{\oint_{\partial D} f(x, y) d x}
$$

provided the denominators are not zero.

For other integral inequalities for multiple integrals see [3]- [15].

In this paper, motivated by the above results and by utilising the famous divergence theorem for $n$-dimensional integrals, we provide some error estimates for approximating the integral on a body $B$, a bounded closed subset of $\mathbb{R}^{n}(n \geq 2)$ with smooth (or piecewise smooth) boundary $\partial B$, by an integral on the surface $\partial B$ and some other simple terms. Some examples for the 3-dimensional case are also given.

2. Some preliminary facts. Let $B$ be a bounded open set in $\mathbb{R}^{n}(n \geq 2)$ with smooth (or piecewise smooth) boundary $\partial B$. Let $F=\left(F_{1}, \ldots, F_{n}\right)$ be a smooth vector field defined in $\mathbb{R}^{n}$, or at least in $B \cup \partial B$. Let $\mathbf{n}$ be the unit outward-pointing normal on $\partial B$. Then the divergence theorem (see for instance [16]) states that

$$
\int_{B} \operatorname{div} F d V=\int_{\partial B} F \cdot \mathbf{n} d A
$$

where

$$
\operatorname{div} F=\nabla \cdot F=\sum_{k=1}^{n} \frac{\partial F_{k}}{\partial x_{k}}
$$

$d V$ is the volume element in $\mathbb{R}^{n}$ and $d A$ is the surface area element on $\partial B$.

If $\mathbf{n}=\left(\mathbf{n}_{1}, \ldots, \mathbf{n}_{n}\right), x=\left(x_{1}, \ldots, x_{n}\right) \in B$ and we write $d x$ for $d V$, we can rewrite (2.1) more explicitly as

$$
\sum_{k=1}^{n} \int_{B} \frac{\partial F_{k}(x)}{\partial x_{k}} d x=\sum_{k=1}^{n} \int_{\partial B} F_{k}(x) n_{k}(x) d A .
$$

By taking the real and imaginary parts, we can extend the above equality to complex-valued functions $F_{k}, k \in\{1, \ldots, n\}$, defined on $B$.

If $n=2$, the normal is obtained by rotating the tangent vector through $90^{\circ}$ (in the correct direction so that it points outwards). The quantity $t d s$ can be written as $\left(d x_{1}, d x_{2}\right)$ along the surface, so that

$$
\mathbf{n} d A:=\mathbf{n} d s=\left(d x_{2},-d x_{1}\right) .
$$

Here $t$ is the tangent vector along the boundary curve and $d s$ is the arc-length element. 
From 2.2 we get, for $B \subset \mathbb{R}^{2}$,

$$
\begin{aligned}
\int_{B} \frac{\partial F_{1}\left(x_{1}, x_{2}\right)}{\partial x_{1}} d x_{1} d x_{2}+ & \int_{B} \frac{\partial F_{2}\left(x_{1}, x_{2}\right)}{\partial x_{2}} d x_{1} d x_{2} \\
& =\int_{\partial B} F_{1}\left(x_{1}, x_{2}\right) d x_{2}-\int_{\partial B} F_{2}\left(x_{1}, x_{2}\right) d x_{1},
\end{aligned}
$$

which is Green's theorem in the plane.

If $n=3$ and if $\partial B$ is described as a level-set of a function of three variables, i.e. $\partial B=\left\{\left(x_{1}, x_{2}, x_{3}\right) \in \mathbb{R}^{3} \mid G\left(x_{1}, x_{2}, x_{3}\right)=0\right\}$, then a vector pointing in the direction of $\mathbf{n}$ is $\operatorname{grad} G$. We shall use the case where $G\left(x_{1}, x_{2}, x_{3}\right)=x_{3}-g\left(x_{1}, x_{2}\right)$ for $\left(x_{1}, x_{2}\right)$ in $D$, a bounded domain in $\mathbb{R}^{2}$ for some differentiable function $g$ on $D$, and

$$
B=\left\{\left(x_{1}, x_{2}, x_{3}\right) \in \mathbb{R}^{3} \mid x_{3}<g\left(x_{1}, x_{2}\right)\right\} .
$$

Also assume that $B$ is bounded in $\mathbb{R}^{3}$. Then

$$
\mathbf{n}=\frac{\left(-g_{x_{1}},-g_{x_{2}}, 1\right)}{\left(1+g_{x_{1}}^{2}+g_{x_{2}}^{2}\right)^{1 / 2}}, \quad d A=\left(1+g_{x_{1}}^{2}+g_{x_{2}}^{2}\right)^{1 / 2} d x_{1} d x_{2}
$$

and

$$
\mathbf{n} d A=\left(-g_{x_{1}},-g_{x_{2}}, 1\right) d x_{1} d x_{2} \text {. }
$$

From 2.2 we get

$$
\begin{aligned}
\int_{B}\left(\frac{\partial F_{1}\left(x_{1}, x_{2}, x_{3}\right)}{\partial x_{1}}+\right. & \left.\frac{\partial F_{2}\left(x_{1}, x_{2}, x_{3}\right)}{\partial x_{2}}+\frac{\partial F_{3}\left(x_{1}, x_{2}, x_{3}\right)}{\partial x_{3}}\right) d x_{1} d x_{2} d x_{3} \\
= & -\int_{D} F_{1}\left(x_{1}, x_{2}, g\left(x_{1}, x_{2}\right)\right) g_{x_{1}}\left(x_{1}, x_{2}\right) d x_{1} d x_{2} \\
& -\int_{D} F_{2}\left(x_{1}, x_{2}, g\left(x_{1}, x_{2}\right)\right) g_{x_{2}}\left(x_{1}, x_{2}\right) d x_{1} d x_{2} \\
& +\int_{D} F_{3}\left(x_{1}, x_{2}, g\left(x_{1}, x_{2}\right)\right) d x_{1} d x_{2}
\end{aligned}
$$

which is the Gauss-Ostrogradsky theorem in space.

Following Apostol [1], consider a surface described by the vector equation

$$
r(u, v)=x_{1}(u, v) \vec{i}+x_{2}(u, v) \vec{j}+x_{3}(u, v) \vec{k}
$$

where $(u, v) \in[a, b] \times[c, d]$.

If $x_{1}, x_{2}, x_{3}$ are differentiable on $[a, b] \times[c, d]$, we consider the two vectors

$$
\begin{aligned}
& \frac{\partial r}{\partial u}=\frac{\partial x_{1}}{\partial u} \vec{i}+\frac{\partial x_{2}}{\partial u} \vec{j}+\frac{\partial x_{3}}{\partial u} \vec{k}, \\
& \frac{\partial r}{\partial v}=\frac{\partial x_{1}}{\partial v} \vec{i}+\frac{\partial x_{2}}{\partial v} \vec{j}+\frac{\partial x_{3}}{\partial v} \vec{k} .
\end{aligned}
$$


Their cross product $\frac{\partial r}{\partial u} \times \frac{\partial r}{\partial v}$ will be referred to as the fundamental vector product of the representation $r$. Its components can be expressed as Jacobian determinants. In fact, we have [1, p. 420]

$$
\begin{aligned}
\frac{\partial r}{\partial u} \times \frac{\partial r}{\partial v} & =\left|\begin{array}{cc}
\frac{\partial x_{2}}{\partial u} & \frac{\partial x_{3}}{\partial u} \\
\frac{\partial x_{2}}{\partial v} & \frac{\partial x_{3}}{\partial v}
\end{array}\right| \vec{i}+\left|\begin{array}{cc}
\frac{\partial x_{3}}{\partial u} & \frac{\partial x_{1}}{\partial u} \\
\frac{\partial x_{3}}{\partial v} & \frac{\partial x_{1}}{\partial v}
\end{array}\right| \vec{j}+\left|\begin{array}{cc}
\frac{\partial x_{1}}{\partial u} & \frac{\partial x_{2}}{\partial u} \\
\frac{\partial x_{1}}{\partial v} & \frac{\partial x_{2}}{\partial v}
\end{array}\right| \vec{k} \\
& =\frac{\partial\left(x_{2}, x_{3}\right)}{\partial(u, v)} \vec{i}+\frac{\partial\left(x_{3}, x_{1}\right)}{\partial(u, v)} \vec{j}+\frac{\partial\left(x_{1}, x_{2}\right)}{\partial(u, v)} \vec{k} .
\end{aligned}
$$

Let $\partial B=r(T)$ be a parametric surface described by a vector-valued function $r$ defined on the box $T=[a, b] \times[c, d]$. The area of $\partial B$ denoted $A_{\partial B}$ is defined by the double integral [1, pp. 424-425]

$$
\begin{aligned}
A_{\partial B} & =\int_{a c}^{b} \int_{c}^{d}\left\|\frac{\partial r}{\partial u} \times \frac{\partial r}{\partial v}\right\| d u d v \\
& =\int_{a c}^{b} \int_{c}^{d} \sqrt{\left(\frac{\partial\left(x_{2}, x_{3}\right)}{\partial(u, v)}\right)^{2}+\left(\frac{\partial\left(x_{3}, x_{1}\right)}{\partial(u, v)}\right)^{2}+\left(\frac{\partial\left(x_{1}, x_{2}\right)}{\partial(u, v)}\right)^{2}} d u d v .
\end{aligned}
$$

We define surface integrals in terms of a parametric representation for the surface. One can prove that under certain general conditions the value of the integral is independent of the representation.

Let $\partial B=r(T)$ be a parametric surface described by a vector-valued differentiable function $r$ defined on $T=[a, b] \times[c, d]$ and let $f: \partial B \rightarrow \mathbb{C}$ be bounded. The surface integral of $f$ over $\partial B$ is defined by [1, p. 430]

$$
\begin{aligned}
\iint_{\partial B} f d A= & \int_{a c}^{b} \int_{c}^{d} f\left(x_{1}, x_{2}, x_{3}\right)\left\|\frac{\partial r}{\partial u} \times \frac{\partial r}{\partial v}\right\| d u d v \\
= & \int_{a c}^{b} \int_{c}^{d} f\left(x_{1}(u, v), x_{2}(u, v), x_{3}(u, v)\right) \\
& \times \sqrt{\left(\frac{\partial\left(x_{2}, x_{3}\right)}{\partial(u, v)}\right)^{2}+\left(\frac{\partial\left(x_{3}, x_{1}\right)}{\partial(u, v)}\right)^{2}+\left(\frac{\partial\left(x_{1}, x_{2}\right)}{\partial(u, v)}\right)^{2}} d u d v .
\end{aligned}
$$

If $\partial B=r(T)$ is a parametric surface, the fundamental vector product $N=\frac{\partial r}{\partial u} \times \frac{\partial r}{\partial v}$ is normal to $\partial B$ at each regular point of the surface. At each such point there are two unit normals, $\mathbf{n}_{1}$ which has the same direction as $N$, and $\mathbf{n}_{2}$ in the opposite direction. Thus

$$
\mathbf{n}_{1}=\frac{N}{\|N\|} \quad \text { and } \quad \mathbf{n}_{2}=-\mathbf{n}_{1} .
$$

Let $\mathbf{n}$ be one of the two normals $\mathbf{n}_{1}$ or $\mathbf{n}_{2}$. Let also $F$ be a vector field defined 
on $\partial B$ and assume that the surface integral

$$
\iint_{\partial B}(F \cdot \mathbf{n}) d A
$$

called the flux surface integral, exists. Here $F \cdot \mathbf{n}$ is the dot or inner product.

We can write [1, p. 434]

$$
\iint_{\partial B}(F \cdot \mathbf{n}) d A= \pm \int_{a c}^{b} \int_{c}^{d} F(r(u, v)) \cdot\left(\frac{\partial r}{\partial u} \times \frac{\partial r}{\partial v}\right) d u d v
$$

where "+" is used if $\mathbf{n}=\mathbf{n}_{1}$, and "-" if $\mathbf{n}=\mathbf{n}_{2}$.

If

$$
F\left(x_{1}, x_{2}, x_{3}\right)=F_{1}\left(x_{1}, x_{2}, x_{3}\right) \vec{i}+F_{2}\left(x_{1}, x_{2}, x_{3}\right) \vec{j}+F_{3}\left(x_{1}, x_{2}, x_{3}\right) \vec{k}
$$

and

$$
r(u, v)=x_{1}(u, v) \vec{i}+x_{2}(u, v) \vec{j}+x_{3}(u, v) \vec{k} \quad \text { where } \quad(u, v) \in[a, b] \times[c, d]
$$

then the flux surface integral for $\mathbf{n}=\mathbf{n}_{1}$ can be explicitly calculated as 1 , p. 435]

$$
\begin{aligned}
\iint_{\partial B}(F \cdot \mathbf{n}) d A= & \int_{a c}^{b} \int_{c}^{d} F_{1}\left(x_{1}(u, v), x_{2}(u, v), x_{3}(u, v)\right) \frac{\partial\left(x_{2}, x_{3}\right)}{\partial(u, v)} d u d v \\
& +\int_{a c}^{b} \int_{c}^{d} F_{2}\left(x_{1}(u, v), x_{2}(u, v), x_{3}(u, v)\right) \frac{\partial\left(x_{3}, x_{1}\right)}{\partial(u, v)} d u d v \\
& +\int_{a c}^{b} \int_{3}^{d} F_{3}\left(x_{1}(u, v), x_{2}(u, v), x_{3}(u, v)\right) \frac{\partial\left(x_{1}, x_{2}\right)}{\partial(u, v)} d u d v .
\end{aligned}
$$

The sum of the double integrals on the right is often written more succinctly as [1, p. 435]

$$
\begin{array}{r}
\iint_{\partial B} F_{1}\left(x_{1}, x_{2}, x_{3}\right) d x_{2} \wedge d x_{3}+\iint_{\partial B} F_{2}\left(x_{1}, x_{2}, x_{3}\right) d x_{3} \wedge d x_{1} \\
\quad+\iint_{\partial B} F_{3}\left(x_{1}, x_{2}, x_{3}\right) d x_{1} \wedge d x_{2} .
\end{array}
$$

Let $B \subset \mathbb{R}^{3}$ be a solid in 3 -space bounded by an orientable closed surface $\partial B$, and let $\mathbf{n}$ be the unit outer normal to $\partial B$. If $F$ is a continuously differentiable vector field defined on $B$, we have the Gauss-Ostrogradsky identity

$$
\iiint_{B}(\operatorname{div} F) d V=\iint_{\partial B}(F \cdot \mathbf{n}) d A
$$

If we express

$$
F\left(x_{1}, x_{2}, x_{3}\right)=F_{1}\left(x_{1}, x_{2}, x_{3}\right) \vec{i}+F_{2}\left(x_{1}, x_{2}, x_{3}\right) \vec{j}+F_{3}\left(x_{1}, x_{2}, x_{3}\right) \vec{k},
$$


then (2.4) can be written as

$$
\begin{gathered}
\iiint_{B}\left(\frac{\partial F_{1}\left(x_{1}, x_{2}, x_{3}\right)}{\partial x_{1}}+\frac{\partial F_{2}\left(x_{1}, x_{2}, x_{3}\right)}{\partial x_{2}}+\frac{\partial F_{3}\left(x_{1}, x_{2}, x_{3}\right)}{\partial x_{3}}\right) d x_{1} d x_{2} d x_{3} \\
=\iint_{\partial B} F_{1}\left(x_{1}, x_{2}, x_{3}\right) d x_{2} \wedge d x_{3}+\iint_{\partial B} F_{2}\left(x_{1}, x_{2}, x_{3}\right) d x_{3} \wedge d x_{1} \\
\quad+\iint_{\partial B} F_{3}\left(x_{1}, x_{2}, x_{3}\right) d x_{1} \wedge d x_{2} .
\end{gathered}
$$

3. Some perturbed identities. For the body $B$ we consider the coordinates of the centre of gravity

$$
G\left(\overline{x_{B, 1}}, \ldots, \overline{x_{B, n}}\right)
$$

defined by

$$
\overline{x_{B, k}}:=\frac{1}{V(B)} \int_{B} x_{k} d x, \quad k \in\{1, \ldots, n\}
$$

where

$$
V(B):=\int_{B} d x
$$

is the volume of $B$.

We have the following identity of interest:

THEOREM 2. Let $B$ be a bounded closed subset of $\mathbb{R}^{n}(n \geq 2)$ with smooth (or piecewise smooth) boundary $\partial B$. Let $f$ be a continuously differentiable function defined in $\mathbb{R}^{n}$, or at least in an open neighbourhood of $B$, and with complex values. If $\alpha_{k}, \beta_{k}, \delta_{k} \in \mathbb{C}$ for $k \in\{1, \ldots, n\}$ with $\sum_{k=1}^{n} \alpha_{k}=1$, then

$$
\begin{aligned}
& \frac{1}{V(B)} \int_{B} f(x) d x=\sum_{k=1}^{n} \frac{1}{V(B)} \int_{B}\left(\beta_{k}-\alpha_{k} x_{k}\right)\left(\frac{\partial f(x)}{\partial x_{k}}-\delta_{k}\right) d x \\
& \quad+\sum_{k=1}^{n} \delta_{k}\left(\beta_{k}-\alpha_{k} \overline{x_{B, k}}\right)+\sum_{k=1}^{n} \frac{1}{V(B)} \int_{\partial B}\left(\alpha_{k} x_{k}-\beta_{k}\right) f(x) n_{k}(x) d A
\end{aligned}
$$

We also have

$$
\begin{aligned}
& \frac{1}{V(B)} \int_{B} f(x) d x \\
& =\sum_{k=1}^{n} \alpha_{k} \frac{1}{V(B)} \int_{B}\left(\gamma_{k}-x_{k}\right)\left(\frac{\partial f(x)}{\partial x_{k}}-\delta_{k}\right) d x \\
& \quad+\sum_{k=1}^{n} \alpha_{k} \delta_{k}\left(\gamma_{k}-\overline{x_{B, k}}\right)+\sum_{k=1}^{n} \alpha_{k} \frac{1}{V(B)} \int_{\partial B}\left(x_{k}-\gamma_{k}\right) f(x) n_{k}(x) d A
\end{aligned}
$$


for all $\gamma_{k} \in \mathbb{C}, k \in\{1, \ldots, n\}$, and in particular

$$
\begin{aligned}
& \frac{1}{V(B)} \int_{B} f(x) d x \\
& =\frac{1}{n} \sum_{k=1}^{n} \frac{1}{V(B)} \int_{B}\left(\gamma_{k}-x_{k}\right)\left(\frac{\partial f(x)}{\partial x_{k}}-\delta_{k}\right) d x \\
& \quad+\frac{1}{n} \sum_{k=1}^{n} \alpha_{k} \delta_{k}\left(\gamma_{k}-\overline{x_{B, k}}\right)+\frac{1}{n} \sum_{k=1}^{n} \frac{1}{V(B)} \int_{\partial B}\left(x_{k}-\gamma_{k}\right) f(x) n_{k}(x) d A .
\end{aligned}
$$

Proof. Let $x=\left(x_{1}, \ldots, x_{n}\right) \in B$. We consider

$$
F_{k}(x)=\left(\alpha_{k} x_{k}-\beta_{k}\right) f(x), \quad k \in\{1, \ldots, n\} .
$$

Then

$$
\frac{\partial F_{k}(x)}{\partial x_{k}}=\alpha_{k} f(x)+\left(\alpha_{k} x_{k}-\beta_{k}\right) \frac{\partial f(x)}{\partial x_{k}}, \quad k \in\{1, \ldots, n\} .
$$

If we sum this equality over $k$ from 1 to $n$ we get

$$
\begin{aligned}
\sum_{k=1}^{n} \frac{\partial F_{k}(x)}{\partial x_{k}} & =\sum_{k=1}^{n} \alpha_{k} f(x)+\sum_{k=1}^{n}\left(\alpha_{k} x_{k}-\beta_{k}\right) \frac{\partial f(x)}{\partial x_{k}} \\
& =f(x)+\sum_{k=1}^{n}\left(\alpha_{k} x_{k}-\beta_{k}\right) \frac{\partial f(x)}{\partial x_{k}}
\end{aligned}
$$

for all $x=\left(x_{1}, \ldots, x_{n}\right) \in B$.

Now, if we integrate (3.4) over $\left(x_{1}, \ldots, x_{n}\right) \in B$ we get

$$
\int_{B}\left(\sum_{k=1}^{n} \frac{\partial F_{k}(x)}{\partial x_{k}}\right) d x=\int_{B} f(x) d x+\sum_{k=1}^{n} \int_{B}\left[\left(\alpha_{k} x_{k}-\beta_{k}\right) \frac{\partial f(x)}{\partial x_{k}}\right] d x .
$$

By the divergence theorem $(2.2)$ we also have

$$
\int_{B}\left(\sum_{k=1}^{n} \frac{\partial F_{k}(x)}{\partial x_{k}}\right) d x=\sum_{k=1}^{n} \int_{\partial B}\left(\alpha_{k} x_{k}-\beta_{k}\right) f(x) n_{k}(x) d A,
$$

and by making use of 3.5 and 3.6 we derive

$$
\begin{aligned}
\int_{B} f(x) d x+\sum_{k=1}^{n} \int_{B}\left[\left(\alpha_{k} x_{k}-\beta_{k}\right) \frac{\partial f(x)}{\partial x_{k}}\right] d x & \\
& =\sum_{k=1}^{n} \int_{\partial B}\left(\alpha_{k} x_{k}-\beta_{k}\right) f(x) n_{k}(x) d A,
\end{aligned}
$$


which gives the representation

$$
\begin{aligned}
\int_{B} f(x) d x= & \sum_{k=1}^{n} \int_{B}\left(\beta_{k}-\alpha_{k} x_{k}\right) \frac{\partial f(x)}{\partial x_{k}} d x \\
& +\sum_{k=1}^{n} \int_{\partial B}\left(\alpha_{k} x_{k}-\beta_{k}\right) f(x) n_{k}(x) d A .
\end{aligned}
$$

Now, observe that

$$
\begin{aligned}
\int_{B}\left(\beta_{k}-\alpha_{k} x_{k}\right) & \left(\frac{\partial f(x)}{\partial x_{k}}-\delta_{k}\right) d x \\
= & \int_{B}\left(\beta_{k}-\alpha_{k} x_{k}\right) \frac{\partial f(x)}{\partial x_{k}} d x-\delta_{k} \int_{B}\left(\beta_{k}-\alpha_{k} x_{k}\right) d x \\
= & \int_{B}\left(\beta_{k}-\alpha_{k} x_{k}\right) \frac{\partial f(x)}{\partial x_{k}} d x-\delta_{k}\left(\beta_{k} V(B)-\alpha_{k} V(B) \overline{x_{B, k}}\right) \\
= & \int_{B}\left(\beta_{k}-\alpha_{k} x_{k}\right) \frac{\partial f(x)}{\partial x_{k}} d x-V(B) \delta_{k}\left(\beta_{k}-\alpha_{k} \overline{x_{B, k}}\right),
\end{aligned}
$$

which for summation over $k \in\{1, \ldots, n\}$ provides

$$
\begin{aligned}
\sum_{k=1}^{n} \int_{B}\left(\beta_{k}-\right. & \left.\alpha_{k} x_{k}\right)\left(\frac{\partial f(x)}{\partial x_{k}}-\delta_{k}\right) d x \\
& =\sum_{k=1}^{n} \int_{B}\left(\beta_{k}-\alpha_{k} x_{k}\right) \frac{\partial f(x)}{\partial x_{k}} d x-V(B) \sum_{k=1}^{n} \delta_{k}\left(\beta_{k}-\alpha_{k} \overline{x_{B, k}}\right),
\end{aligned}
$$

and so

$$
\begin{aligned}
& \sum_{k=1}^{n} \int_{B}\left(\beta_{k}-\alpha_{k} x_{k}\right) \frac{\partial f(x)}{\partial x_{k}} d x \\
& \quad=\sum_{k=1}^{n} \int_{B}\left(\beta_{k}-\alpha_{k} x_{k}\right)\left(\frac{\partial f(x)}{\partial x_{k}}-\delta_{k}\right) d x+V(B) \sum_{k=1}^{n} \delta_{k}\left(\beta_{k}-\alpha_{k} \overline{x_{B, k}}\right) .
\end{aligned}
$$

From (3.7) we then get

$$
\begin{aligned}
\int_{B} f(x) d x= & \sum_{k=1}^{n} \int_{B}\left(\beta_{k}-\alpha_{k} x_{k}\right)\left(\frac{\partial f(x)}{\partial x_{k}}-\delta_{k}\right) d x \\
& +V(B) \sum_{k=1}^{n} \delta_{k}\left(\beta_{k}-\alpha_{k} \overline{x_{B, k}}\right)+\sum_{k=1}^{n} \int_{\partial B}\left(\alpha_{k} x_{k}-\beta_{k}\right) f(x) n_{k}(x) d A,
\end{aligned}
$$

which on division by $V(B)$ produces the desired result (3.1).

The identity (3.2) follows from (3.1) for $\beta_{k}=\alpha_{k} \gamma_{k}, k \in\{1, \ldots, n\}$. 
The following particular cases are of interest:

Corollary 1. With the assumptions of Theorem 2 we have

$$
\begin{aligned}
\frac{1}{V(B)} \int_{B} f(x) d x= & \sum_{k=1}^{n} \alpha_{k} \frac{1}{V(B)} \int_{B}\left(\overline{x_{B, k}}-x_{k}\right)\left(\frac{\partial f(x)}{\partial x_{k}}-\delta_{k}\right) d x \\
& +\sum_{k=1}^{n} \alpha_{k} \frac{1}{V(B)} \int_{\partial B}\left(x_{k}-\overline{x_{B, k}}\right) f(x) n_{k}(x) d A,
\end{aligned}
$$

and in particular

$$
\begin{aligned}
\frac{1}{V(B)} \int_{B} f(x) d x= & \frac{1}{n} \sum_{k=1}^{n} \frac{1}{V(B)} \int_{B}\left(\overline{x_{B, k}}-x_{k}\right)\left(\frac{\partial f(x)}{\partial x_{k}}-\delta_{k}\right) d x \\
& +\frac{1}{n} \sum_{k=1}^{n} \frac{1}{V(B)} \int_{\partial B}\left(x_{k}-\overline{x_{B, k}}\right) f(x) n_{k}(x) d A .
\end{aligned}
$$

This follows from (3.1) on taking $\beta_{k}=\alpha_{k} \overline{x_{B, k}}, k \in\{1, \ldots, n\}$.

For a function $f$ as in Theorem 2 above, we define the points

$$
x_{\partial B, f, k}:=\frac{\int_{\partial B} x_{k} f(x) n_{k}(x) d A}{\int_{\partial B} f(x) n_{k}(x) d A}, \quad k \in\{1, \ldots, n\},
$$

provided that all denominators are nonzero.

Corollary 2. With the assumptions of Theorem 2 we have

$$
\begin{aligned}
\frac{1}{V(B)} \int_{B} f(x) d x= & \sum_{k=1}^{n} \alpha_{k} \frac{1}{V(B)} \int_{B}\left(x_{\partial B, f, k}-x_{k}\right)\left(\frac{\partial f(x)}{\partial x_{k}}-\delta_{k}\right) d x \\
& +\sum_{k=1}^{n} \delta_{k} \alpha_{k}\left(x_{\partial B, f, k}-\overline{x_{B, k}}\right)
\end{aligned}
$$

and in particular

$$
\begin{aligned}
\frac{1}{V(B)} \int_{B} f(x) d x= & \frac{1}{n} \sum_{k=1}^{n} \frac{1}{V(B)} \int_{B}\left(x_{\partial B, f, k}-x_{k}\right)\left(\frac{\partial f(x)}{\partial x_{k}}-\delta_{k}\right) d x \\
& +\frac{1}{n} \sum_{k=1}^{n} \delta_{k}\left(x_{\partial B, f, k}-\overline{x_{B, k}}\right) .
\end{aligned}
$$

This follows from (3.1) on taking $\beta_{k}=\alpha_{k} x_{\partial B, f, k}, k \in\{1, \ldots, n\}$, and observing that

$$
\sum_{k=1}^{n} \alpha_{k} \int_{\partial B}\left(x_{k}-x_{\partial B, f, k}\right) f(x) n_{k}(x) d A=0 .
$$


4. Some inequalities for bounded partial derivatives. Let $B$ be a bounded closed subset of $\mathbb{R}^{n}(n \geq 2)$ with smooth (or piecewise smooth) boundary $\partial B$. Now, for $\phi, \Phi \in \mathbb{C}$, define the following sets of complex-valued functions:

$$
\begin{aligned}
& \bar{U}_{B}(\phi, \Phi):=\{f: B \rightarrow \mathbb{C} \mid \operatorname{Re}[(\Phi-f(x))(\overline{f(x)}-\bar{\phi})] \geq 0 \text { for each } x \in B\}, \\
& \bar{\Delta}_{B}(\phi, \Phi):=\left\{f: B \rightarrow \mathbb{C}|| f(x)-\frac{\phi+\Phi}{2}\left|\leq \frac{1}{2}\right| \Phi-\phi \mid \text { for each } x \in B\right\} .
\end{aligned}
$$

The following representation result may be stated.

Proposition 1. For any distinct $\phi, \Phi \in \mathbb{C}$, the sets $\bar{U}_{B}(\phi, \Phi)$ and $\bar{\Delta}_{B}(\phi, \Phi)$ are nonempty, convex and closed and

$$
\bar{U}_{B}(\phi, \Phi)=\bar{\Delta}_{B}(\phi, \Phi) .
$$

Proof. We observe that for any $w \in \mathbb{C}$ we have the equivalence

$$
\left|w-\frac{\phi+\Phi}{2}\right| \leq \frac{1}{2}|\Phi-\phi| \Longleftrightarrow \operatorname{Re}[(\Phi-w)(\bar{w}-\bar{\phi})] \geq 0 .
$$

This follows from the equality

$$
\frac{1}{4}|\Phi-\phi|^{2}-\left|w-\frac{\phi+\Phi}{2}\right|^{2}=\operatorname{Re}[(\Phi-w)(\bar{w}-\bar{\phi})],
$$

which holds for any $w \in \mathbb{C}$.

The equality (4.1) is thus a simple consequence of this fact.

On making use of the properties of complex numbers we can also state that:

Corollary 3. For any distinct $\phi, \Phi \in \mathbb{C}$, we have

$$
\begin{aligned}
\bar{U}_{B}(\phi, \Phi) & =\{f: B \rightarrow \mathbb{C} \mid(\operatorname{Re} \Phi-\operatorname{Re} f(x))(\operatorname{Re} f(x)-\operatorname{Re} \phi) \\
& +(\operatorname{Im} \Phi-\operatorname{Im} f(x))(\operatorname{Im} f(x)-\operatorname{Im} \phi) \geq 0 \text { for each } x \in B\} .
\end{aligned}
$$

Now, if we assume that $\operatorname{Re}(\Phi) \geq \operatorname{Re}(\phi)$ and $\operatorname{Im}(\Phi) \geq \operatorname{Im}(\phi)$, then we can define the following set of functions as well:

$$
\begin{aligned}
\bar{S}_{B}(\phi, \Phi):=\{f: B \rightarrow \mathbb{C} \mid & \operatorname{Re} \Phi \geq \operatorname{Re} f(x) \geq \operatorname{Re} \phi \text { and } \\
& \operatorname{Im} \Phi \geq \operatorname{Im} f(x) \geq \operatorname{Im} \phi \text { for each } x \in B\} .
\end{aligned}
$$

One can easily observe that $\bar{S}_{B}(\phi, \Phi)$ is closed, convex and

$$
\emptyset \neq \bar{S}_{B}(\phi, \Phi) \subseteq \bar{U}_{B}(\phi, \Phi) .
$$

TheOREM 3. Let $B$ be a bounded closed subset of $\mathbb{R}^{n}(n \geq 2)$ with smooth (or piecewise smooth) boundary $\partial B$. Let $f$ be a continuously differentiable function defined in $\mathbb{R}^{n}$, or at least in an open neighbourhood of $B$ and with complex values. Assume that there exist $\phi_{k}, \Phi_{k} \in \mathbb{C}, \phi_{k} \neq \Phi_{k}$ 
for $k \in\{1, \ldots, n\}$ and such that $\frac{\partial f}{\partial x_{k}} \in \bar{\Delta}_{B}\left(\phi_{k}, \Phi_{k}\right)$ for $k \in\{1, \ldots, n\}$. If $\alpha_{k}, \beta_{k} \in \mathbb{C}$ for $k \in\{1, \ldots, n\}$ with $\sum_{k=1}^{n} \alpha_{k}=1$, then

$$
\begin{aligned}
\mid \frac{1}{V(B)} \int_{B} f(x) d x-\sum_{k=1}^{n} \frac{\phi_{k}+\Phi_{k}}{2}\left(\beta_{k}-\alpha_{k} \overline{x_{B, k}}\right) \\
\quad-\sum_{k=1}^{n} \frac{1}{V(B)} \int_{\partial B}\left(\alpha_{k} x_{k}-\beta_{k}\right) f(x) n_{k}(x) d A \mid \\
\leq \frac{1}{2} \sum_{k=1}^{n}\left|\Phi_{k}-\phi_{k}\right| \frac{1}{V(B)} \int_{B}\left|\beta_{k}-\alpha_{k} x_{k}\right| d x .
\end{aligned}
$$

We also have

$$
\begin{aligned}
\mid \frac{1}{V(B)} \int_{B} f(x) d x-\sum_{k=1}^{n} \frac{\phi_{k}+\Phi_{k}}{2} \alpha_{k}\left(\gamma_{k}-\overline{x_{B, k}}\right) \\
\quad-\sum_{k=1}^{n} \alpha_{k} \frac{1}{V(B)} \int_{\partial B}\left(x_{k}-\gamma_{k}\right) f(x) n_{k}(x) d A \mid \\
\leq \frac{1}{2} \sum_{k=1}^{n}\left|\Phi_{k}-\phi_{k}\right|\left|\alpha_{k}\right| \frac{1}{V(B)} \int_{B}\left|\gamma_{k}-x_{k}\right| d x
\end{aligned}
$$

for all $\gamma_{k} \in \mathbb{C}, k \in\{1, \ldots, n\}$, and in particular

$$
\begin{aligned}
\mid \frac{1}{V(B)} \int_{B} f(x) d x-\frac{1}{n} \sum_{k=1}^{n} \frac{\phi_{k}+\Phi_{k}}{2}\left(\gamma_{k}-\overline{x_{B, k}}\right) \\
\quad-\frac{1}{n} \sum_{k=1}^{n} \frac{1}{V(B)} \int_{\partial B}\left(x_{k}-\gamma_{k}\right) f(x) n_{k}(x) d A \mid \\
\leq \frac{1}{2 n} \sum_{k=1}^{n}\left|\Phi_{k}-\phi_{k}\right| \frac{1}{V(B)} \int_{B}\left|\gamma_{k}-x_{k}\right| d x .
\end{aligned}
$$

Proof. By using identity 3.1 for $\delta_{k}:=\frac{\phi_{k}+\Phi_{k}}{2}, k \in\{1, \ldots, n\}$, we get

$$
\text { 8) } \begin{aligned}
& \frac{1}{V(B)} \int_{B} f(x) d x \\
= & \sum_{k=1}^{n} \frac{1}{V(B)} \int_{B}\left(\beta_{k}-\alpha_{k} x_{k}\right)\left(\frac{\partial f(x)}{\partial x_{k}}-\frac{\phi_{k}+\Phi_{k}}{2}\right) d x \\
+ & \sum_{k=1}^{n} \frac{\phi_{k}+\Phi_{k}}{2}\left(\beta_{k}-\alpha_{k} \overline{x_{B, k}}\right)+\sum_{k=1}^{n} \frac{1}{V(B)} \int_{\partial B}\left(\alpha_{k} x_{k}-\beta_{k}\right) f(x) n_{k}(x) d A .
\end{aligned}
$$

Since $\frac{\partial f}{\partial x_{k}} \in \bar{\Delta}_{B}\left(\phi_{k}, \Phi_{k}\right)$ for $k \in\{1, \ldots, n\}, 4.8$ yields 


$$
\begin{aligned}
\mid \frac{1}{V(B)} \int_{B} f(x) d x \leq & \sum_{k=1}^{n} \frac{\phi_{k}+\Phi_{k}}{2}\left(\beta_{k}-\alpha_{k} \overline{x_{B, k}}\right) \\
& -\sum_{k=1}^{n} \frac{1}{V(B)} \int_{\partial B}\left(\alpha_{k} x_{k}-\beta_{k}\right) f(x) n_{k}(x) d A \mid \\
\leq & \sum_{k=1}^{n} \frac{1}{V(B)}\left|\int_{B}\left(\beta_{k}-\alpha_{k} x_{k}\right)\left(\frac{\partial f(x)}{\partial x_{k}}-\frac{\phi_{k}+\Phi_{k}}{2}\right) d x\right| \\
\leq & \sum_{k=1}^{n} \frac{1}{V(B)} \int_{B}\left|\left(\beta_{k}-\alpha_{k} x_{k}\right)\left(\frac{\partial f(x)}{\partial x_{k}}-\frac{\phi_{k}+\Phi_{k}}{2}\right)\right| d x \\
\leq & \sum_{k=1}^{n} \frac{1}{2}\left|\Phi_{k}-\phi_{k}\right| \frac{1}{V(B)} \int_{B}\left|\beta_{k}-\alpha_{k} x_{k}\right| d x
\end{aligned}
$$

which proves 4.5 . The rest is obvious.

COROLlary 4. With the assumptions of Theorem 3 we have

$$
\begin{aligned}
\mid \frac{1}{V(B)} \int_{B} f(x) d x- & \sum_{k=1}^{n} \alpha_{k} \frac{1}{V(B)} \int_{\partial B}\left(x_{k}-\overline{x_{B, k}}\right) f(x) n_{k}(x) d A \mid \\
& \leq \frac{1}{2} \sum_{k=1}^{n}\left|\alpha_{k}\right|\left|\Phi_{k}-\phi_{k}\right| \frac{1}{V(B)} \int_{B}\left|\overline{x_{B, k}}-x_{k}\right| d x
\end{aligned}
$$

and in particular

$$
\begin{array}{r}
\left|\frac{1}{V(B)} \int_{B} f(x) d x-\frac{1}{n} \sum_{k=1}^{n} \frac{1}{V(B)} \int_{\partial B}\left(x_{k}-\overline{x_{B, k}}\right) f(x) n_{k}(x) d A\right| \\
\leq \frac{1}{2 n} \sum_{k=1}^{n}\left|\Phi_{k}-\phi_{k}\right| \frac{1}{V(B)} \int_{B}\left|\overline{x_{B, k}}-x_{k}\right| d x .
\end{array}
$$

This follows from (4.6) by taking $\gamma_{k}=\overline{x_{B, k}}, k \in\{1, \ldots, n\}$.

Corollary 5. With the assumptions of Theorem 3 we have

$$
\begin{aligned}
\mid \frac{1}{V(B)} \int_{B} f(x) d x & -\sum_{k=1}^{n} \frac{\phi_{k}+\Phi_{k}}{2} \alpha_{k}\left(x_{\partial B, f, k}-\overline{x_{B, k}}\right) \mid \\
& \leq \frac{1}{2} \sum_{k=1}^{n}\left|\Phi_{k}-\phi_{k}\right|\left|\alpha_{k}\right| \frac{1}{V(B)} \int_{B}\left|x_{\partial B, f, k}-x_{k}\right| d x
\end{aligned}
$$

and in particular 


$$
\begin{aligned}
\mid \frac{1}{V(B)} \int_{B} f(x) d x- & \frac{1}{n} \sum_{k=1}^{n} \frac{\phi_{k}+\Phi_{k}}{2}\left(x_{\partial B, f, k}-\overline{x_{B, k}}\right) \mid \\
& \leq \frac{1}{2 n} \sum_{k=1}^{n}\left|\Phi_{k}-\phi_{k}\right| \frac{1}{V(B)} \int_{B}\left|x_{\partial B, f, k}-x_{k}\right| d x .
\end{aligned}
$$

This follows from (4.6) by taking $\gamma_{k}=x_{\partial B, f, k}, k \in\{1, \ldots, n\}$, and observing that

$$
\sum_{k=1}^{n} \alpha_{k} \frac{1}{V(B)} \int_{\partial B}\left(x_{k}-x_{\partial B, f, k}\right) f(x) n_{k}(x) d A=0 .
$$

5. Inequalities for Lipschitzian partial derivatives. We assume that the partial derivatives $\frac{\partial f}{\partial x_{k}}, k \in\{1, \ldots, n\}$, satisfy the Lipschitz type conditions at the point $u=\left(u_{1}, \ldots, u_{n}\right) \in D$ :

$$
\left|\frac{\partial f(x)}{\partial x_{k}}-\frac{\partial f(u)}{\partial x_{k}}\right| \leq \sum_{j=1}^{n} L_{k, j}\left|x_{j}-u_{j}\right|
$$

for any $x=\left(x_{1}, \ldots, x_{n}\right) \in D$, where $L_{k, j}, k, j \in\{1, \ldots, n\}$, are given positive constants.

THEOREM 4. Let $B$ be a bounded closed subset of $\mathbb{R}^{n}(n \geq 2)$ with smooth (or piecewise smooth) boundary $\partial B$. Let $f$ be a continuously differentiable function defined in $\mathbb{R}^{n}$, or at least in an open neighbourhood of $B$, and with complex values. Assume that for $u \in B$ there exist $L_{k, j}, k, j \in\{1, \ldots, n\}$, such that the Lipschitz condition (5.1) holds for $k \in\{1, \ldots, n\}$. If $\alpha_{k}, \beta_{k} \in \mathbb{C}$ for $k \in\{1, \ldots, n\}$ with $\sum_{k=1}^{n} \alpha_{k}=1$, then

We also have

$$
\begin{aligned}
\mid \frac{1}{V(B)} \int_{B} f(x) d x- & \sum_{k=1}^{n} \frac{\partial f(u)}{\partial x_{k}}\left(\beta_{k}-\alpha_{k} \overline{x_{B, k}}\right) \\
& -\sum_{k=1}^{n} \frac{1}{V(B)} \int_{\partial B}\left(\alpha_{k} x_{k}-\beta_{k}\right) f(x) n_{k}(x) d A \mid \\
\leq & \sum_{k=1}^{n} \sum_{j=1}^{n} L_{k, j} \frac{1}{V(B)} \int_{B}\left|\beta_{k}-\alpha_{k} x_{k}\right|\left|x_{j}-u_{j}\right| d x .
\end{aligned}
$$

$$
\begin{aligned}
\mid \frac{1}{V(B)} \int_{B} f(x) d x & -\sum_{k=1}^{n} \alpha_{k} \frac{\partial f(u)}{\partial x_{k}}\left(\gamma_{k}-\overline{x_{B, k}}\right) \\
& -\sum_{k=1}^{n} \frac{1}{V(B)} \int_{\partial B} \alpha_{k}\left(x_{k}-\gamma_{k}\right) f(x) n_{k}(x) d A \mid \\
\leq & \sum_{k=1}^{n} \sum_{j=1}^{n} L_{k, j}\left|\alpha_{k}\right| \frac{1}{V(B)} \int_{B}\left|\gamma_{k}-x_{k}\right|\left|x_{j}-u_{j}\right| d x
\end{aligned}
$$


for all $\gamma_{k} \in \mathbb{C}, k \in\{1, \ldots, n\}$, and in particular

$$
\begin{aligned}
\mid \frac{1}{V(B)} \int_{B} f(x) d x- & \frac{1}{n} \sum_{k=1}^{n} \frac{\partial f(u)}{\partial x_{k}}\left(\gamma_{k}-\overline{x_{B, k}}\right) \\
& -\frac{1}{n} \sum_{k=1}^{n} \frac{1}{V(B)} \int_{\partial B} \alpha_{k}\left(x_{k}-\gamma_{k}\right) f(x) n_{k}(x) d A \mid \\
\leq & \frac{1}{n} \sum_{k=1}^{n} \sum_{j=1}^{n} L_{k, j} \frac{1}{V(B)} \int_{B}\left|\gamma_{k}-x_{k}\right|\left|x_{j}-u_{j}\right| d x .
\end{aligned}
$$

Proof. If we write the equality 3.1 for $\delta_{k}=\frac{\partial f(u)}{\partial x_{k}}, k \in\{1, \ldots, n\}$, we obtain

$$
\begin{aligned}
& \frac{1}{V(B)} \int_{B} f(x) d x=\sum_{k=1}^{n} \frac{1}{V(B)} \int_{B}\left(\beta_{k}-\alpha_{k} x_{k}\right)\left(\frac{\partial f(x)}{\partial x_{k}}-\frac{\partial f(u)}{\partial x_{k}}\right) d x \\
& \quad+\sum_{k=1}^{n} \frac{\partial f(u)}{\partial x_{k}}\left(\beta_{k}-\alpha_{k} \overline{x_{B, k}}\right)+\sum_{k=1}^{n} \frac{1}{V(B)} \int_{\partial B}\left(\alpha_{k} x_{k}-\beta_{k}\right) f(x) n_{k}(x) d A
\end{aligned}
$$

Therefore

$$
\begin{aligned}
\mid \frac{1}{V(B)} \int_{B} f(x) d x- & \sum_{k=1}^{n} \frac{\partial f(u)}{\partial x_{k}}\left(\beta_{k}-\alpha_{k} \overline{x_{B, k}}\right) \\
& -\sum_{k=1}^{n} \frac{1}{V(B)} \int_{\partial B}\left(\alpha_{k} x_{k}-\beta_{k}\right) f(x) n_{k}(x) d A \mid \\
\leq & \sum_{k=1}^{n} \frac{1}{V(B)}\left|\int_{B}\left(\beta_{k}-\alpha_{k} x_{k}\right)\left(\frac{\partial f(x)}{\partial x_{k}}-\frac{\partial f(u)}{\partial x_{k}}\right) d x\right| \\
\leq & \sum_{k=1}^{n} \frac{1}{V(B)} \int_{B}\left|\left(\beta_{k}-\alpha_{k} x_{k}\right)\left(\frac{\partial f(x)}{\partial x_{k}}-\frac{\partial f(u)}{\partial x_{k}}\right)\right| d x \\
= & \sum_{k=1}^{n} \frac{1}{V(B)} \int_{B}\left|\beta_{k}-\alpha_{k} x_{k}\right|\left|\frac{\partial f(x)}{\partial x_{k}}-\frac{\partial f(u)}{\partial x_{k}}\right| d x \\
\leq & \sum_{k=1}^{n} \frac{1}{V(B)} \int_{B}\left|\beta_{k}-\alpha_{k} x_{k}\right| \sum_{j=1}^{n} L_{k, j}\left|x_{j}-u_{j}\right| d x \\
= & \sum_{k=1}^{n} \sum_{j=1}^{n} L_{k, j} \frac{1}{V(B)} \int_{B}\left|\beta_{k}-\alpha_{k} x_{k}\right|\left|x_{j}-u_{j}\right| d x
\end{aligned}
$$

and the inequality 5.2 is proved. 
Corollary 6. With the assumptions of Theorem 4 we have

$$
\begin{aligned}
\mid \frac{1}{V(B)} \int_{B} f(x) d x & -\sum_{k=1}^{n} \frac{1}{V(B)} \int_{\partial B} \alpha_{k}\left(x_{k}-\overline{x_{B, k}}\right) f(x) n_{k}(x) d A \mid \\
\leq & \sum_{k=1}^{n} \sum_{j=1}^{n} L_{k, j}\left|\alpha_{k}\right| \frac{1}{V(B)} \int_{B}\left|\overline{x_{B, k}}-x_{k}\right|\left|x_{j}-u_{j}\right| d x
\end{aligned}
$$

and in particular

$$
\begin{aligned}
\mid \frac{1}{V(B)} \int_{B} f(x) d x & -\frac{1}{n} \sum_{k=1}^{n} \frac{1}{V(B)} \int_{\partial B}\left(x_{k}-\overline{x_{B, k}}\right) f(x) n_{k}(x) d A \mid \\
& \leq \frac{1}{n} \sum_{k=1}^{n} \sum_{j=1}^{n} L_{k, j} \frac{1}{V(B)} \int_{B}\left|\overline{x_{B, k}}-x_{k}\right|\left|x_{j}-u_{j}\right| d x .
\end{aligned}
$$

COROLlary 7. With the assumptions of Theorem 4 we have

$$
\begin{aligned}
& \mid \frac{1}{V(B)} \int_{B} f(x) d x-\sum_{k=1}^{n} \alpha_{k} \frac{\partial f(u)}{\partial x_{k}}\left(x_{\partial B, f, k}-\overline{x_{B, k}}\right) \mid \\
& \leq \sum_{k=1}^{n} \sum_{j=1}^{n} L_{k, j}\left|\alpha_{k}\right| \frac{1}{V(B)} \int_{B}\left|x_{\partial B, f, k}-x_{k}\right|\left|x_{j}-u_{j}\right| d x,
\end{aligned}
$$

and in particular

$$
\begin{aligned}
\mid \frac{1}{V(B)} \int_{B} f(x) & d x-\frac{1}{n} \sum_{k=1}^{n} \frac{\partial f(u)}{\partial x_{k}}\left(x_{\partial B, f, k}-\overline{x_{B, k}}\right) \mid \\
\leq & \frac{1}{n} \sum_{k=1}^{n} \sum_{j=1}^{n} L_{k, j} \frac{1}{V(B)} \int_{B}\left|x_{\partial B, f, k}-x_{k}\right|\left|x_{j}-u_{j}\right| d x .
\end{aligned}
$$

COROLlary 8. With the assumptions of Theorem 4 we have

$$
\begin{aligned}
\mid \frac{1}{V(B)} \int_{B} f(x) d x & -\sum_{k=1}^{n} \alpha_{k} \frac{\partial f(u)}{\partial x_{k}}\left(u_{k}-\overline{x_{B, k}}\right) \\
& -\sum_{k=1}^{n} \frac{1}{V(B)} \int_{\partial B} \alpha_{k}\left(x_{k}-u_{k}\right) f(x) n_{k}(x) d A \mid \\
\leq & \sum_{k=1}^{n} \sum_{j=1}^{n} L_{k, j}\left|\alpha_{k}\right| \frac{1}{V(B)} \int_{B}\left|u_{k}-x_{k}\right|\left|x_{j}-u_{j}\right| d x
\end{aligned}
$$


and in particular

$$
\begin{aligned}
\mid \frac{1}{V(B)} \int_{B} f(x) d x & -\frac{1}{n} \sum_{k=1}^{n} \frac{\partial f(u)}{\partial x_{k}}\left(u_{k}-\overline{x_{B, k}}\right) \\
& -\frac{1}{n} \sum_{k=1}^{n} \frac{1}{V(B)} \int_{\partial B}\left(x_{k}-u_{k}\right) f(x) n_{k}(x) d A \mid \\
\leq & \frac{1}{n} \sum_{k=1}^{n} \sum_{j=1}^{n} L_{k, j} \frac{1}{V(B)} \int_{B}\left|u_{k}-x_{k}\right|\left|x_{j}-u_{j}\right| d x .
\end{aligned}
$$

REMARK 1. With the assumptions of Theorem 4 and for $G=$ $\left(\overline{x_{B, 1}}, \ldots, \overline{x_{B, n}}\right) \in B$ if there exist $M_{k, j}>0, k, j \in\{1, \ldots, n\}$, such that the Lipschitz conditions

$$
\left|\frac{\partial f(x)}{\partial x_{k}}-\frac{\partial f(G)}{\partial x_{k}}\right| \leq \sum_{j=1}^{n} M_{k, j}\left|x_{j}-u_{j}\right|
$$

hold for $k \in\{1, \ldots, n\}$, then

$$
\begin{aligned}
\left|\frac{1}{V(B)} \int_{B} f(x) d x-\sum_{k=1}^{n} \frac{1}{V(B)} \int_{\partial B} \alpha_{k}\left(x_{k}-\overline{x_{B, k}}\right) f(x) n_{k}(x) d A\right| \\
\leq \sum_{k=1}^{n} \sum_{j=1}^{n} L_{k, j}\left|\alpha_{k}\right| \frac{1}{V(B)} \int_{B}\left|\overline{x_{B, k}}-x_{k}\right|\left|x_{j}-\overline{x_{B, j}}\right| d x
\end{aligned}
$$

and in particular

$$
\begin{aligned}
\mid \frac{1}{V(B)} \int_{B} f(x) & d x-\frac{1}{n} \sum_{k=1}^{n} \frac{1}{V(B)} \int_{\partial B}\left(x_{k}-\overline{x_{B, k}}\right) f(x) n_{k}(x) d A \mid \\
\leq & \frac{1}{n} \sum_{k=1}^{n} \sum_{j=1}^{n} L_{k, j} \frac{1}{V(B)} \int_{B}\left|\overline{x_{B, k}}-x_{k}\right|\left|x_{j}-\overline{x_{B, j}}\right| d x .
\end{aligned}
$$

It is well known that if a function $g$ has bounded partial derivatives on $B$, which is assumed also to be convex, then for all $x, y \in B$ we have the Lipschitz type condition

$$
|g(x)-g(y)| \leq \sum_{j=1}^{n}\left\|\frac{\partial g}{\partial x_{j}}\right\|_{B, \infty}\left|x_{j}-y_{j}\right|
$$

where

$$
\left\|\frac{\partial g}{\partial x_{j}}\right\|_{B, \infty}:=\sup _{x \in B}\left|\frac{\partial g(x)}{\partial x_{j}}\right|<\infty .
$$

We can state the following result that is more convenient to apply: 
Corollary 9. Let $B$ be a bounded closed convex subset of $\mathbb{R}^{n}(n \geq 2)$ with smooth (or piecewise smooth) boundary $\partial B$. Let $f$ be a twice differentiable function defined in $\mathbb{R}^{n}$, or at least in an open neighbourhood of $B$, and with complex values and assume that

$$
\left\|\frac{\partial^{2} f}{\partial x_{k} \partial x_{j}}\right\|_{B, \infty}:=\sup _{x \in B}\left|\frac{\partial^{2} f(x)}{\partial x_{k} \partial x_{j}}\right|<\infty
$$

for all $k, j \in\{1, \ldots, n\}$. Then for all $u \in B$ we have

$$
\begin{aligned}
\mid \frac{1}{V(B)} \int_{B} f(x) d x-\sum_{k=1}^{n} \alpha_{k} \frac{\partial f(u)}{\partial x_{k}}\left(u_{k}-\overline{x_{B, k}}\right) \\
\quad-\sum_{k=1}^{n} \frac{1}{V(B)} \int_{\partial B} \alpha_{k}\left(x_{k}-u_{k}\right) f(x) n_{k}(x) d A \mid \\
\leq \sum_{k=1}^{n} \sum_{j=1}^{n}\left\|\frac{\partial^{2} f}{\partial x_{k} \partial x_{j}}\right\|_{B, \infty}\left|\alpha_{k}\right| \frac{1}{V(B)} \int_{B}\left|u_{k}-x_{k}\right|\left|x_{j}-u_{j}\right| d x
\end{aligned}
$$

and in particular

$$
\begin{array}{rl}
\mid \frac{1}{V(B)} \int_{B} & f(x) d x-\frac{1}{n} \sum_{k=1}^{n} \frac{\partial f(u)}{\partial x_{k}}\left(u_{k}-\overline{x_{B, k}}\right) \\
& -\frac{1}{n} \sum_{k=1}^{n} \frac{1}{V(B)} \int_{\partial B}\left(x_{k}-u_{k}\right) f(x) n_{k}(x) d A \mid \\
\leq & \frac{1}{n} \sum_{k=1}^{n} \sum_{j=1}^{n}\left\|\frac{\partial^{2} f}{\partial x_{k} \partial x_{j}}\right\|_{B, \infty} \frac{1}{V(B)} \int_{B}\left|u_{k}-x_{k}\right|\left|x_{j}-u_{j}\right| d x .
\end{array}
$$

We also have the centre of gravity inequality

$$
\begin{aligned}
& \left|\frac{1}{V(B)} \int_{B} f(x) d x-\sum_{k=1}^{n} \frac{1}{V(B)} \int_{\partial B} \alpha_{k}\left(x_{k}-\overline{x_{B, k}}\right) f(x) n_{k}(x) d A\right| \\
& \leq \sum_{k=1}^{n} \sum_{j=1}^{n}\left|\alpha_{k}\right| \|\left.\frac{\partial^{2} f}{\partial x_{k} \partial x_{j}}\right|_{B, \infty} \frac{1}{V(B)} \int_{B}\left|\overline{x_{B, k}}-x_{k}\right|\left|x_{j}-\overline{x_{B, k}}\right| d x,
\end{aligned}
$$

and in particular

$$
\begin{aligned}
& \left|\frac{1}{V(B)} \int_{B} f(x) d x-\frac{1}{n} \sum_{k=1}^{n} \frac{1}{V(B)} \int_{\partial B}\left(x_{k}-\overline{x_{B, k}}\right) f(x) n_{k}(x) d A\right| \\
& \quad \leq \frac{1}{n} \sum_{k=1}^{n} \sum_{j=1}^{n} \|\left.\frac{\partial^{2} f}{\partial x_{k} \partial x_{j}}\right|_{B, \infty} \frac{1}{V(B)} \int_{B}\left|\overline{x_{B, k}}-x_{k}\right|\left|x_{j}-\overline{x_{B, k}}\right| d x .
\end{aligned}
$$


6. Example for 3-dimensional spaces. Let $B$ be a bounded closed convex subset of $\mathbb{R}^{3}$ with smooth (or piecewise smooth) boundary $\partial B$. Let $f$ be a twice differentiable function defined in $\mathbb{R}^{3}$, or at least in an open neighbourhood of $B$, and with complex values and assume that

$$
\left\|\frac{\partial^{2} f}{\partial x_{k} \partial x_{j}}\right\|_{B, \infty}:=\sup _{x \in B}\left|\frac{\partial^{2} f(x)}{\partial x_{k} \partial x_{j}}\right|<\infty \quad \text { for all } k, j \in\{1,2,3\} .
$$

Assume that $\partial B$ is described by the vector equation

$$
r(u, v)=x_{1}(u, v) \vec{i}+x_{2}(u, v) \vec{j}+x_{3}(u, v) \vec{k}
$$

where $(u, v) \in[a, b] \times[c, d]$. Then, by using the notations from the second section, we have

$$
\begin{aligned}
& \text { 1) } \mid \frac{1}{V(B)} \int_{B} f(x) d x-\sum_{k=1}^{3} \alpha_{k} \frac{\partial f\left(y_{1}, y_{2}, y_{3}\right)}{\partial x_{k}}\left(y_{k}-\overline{x_{B, k}}\right) \\
& -\frac{1}{V(B)} \int_{a c}^{b} \int_{c}^{d} \alpha_{1}\left(x_{1}(u, v)-y_{1}\right) f\left(x_{1}(u, v), x_{2}(u, v), x_{3}(u, v)\right) \frac{\partial\left(x_{2}, x_{3}\right)}{\partial(u, v)} d u d v \\
& -\frac{1}{V(B)} \int_{a c}^{b d} \int_{2} \alpha_{2}\left(x_{2}(u, v)-y_{2}\right) f\left(x_{1}(u, v), x_{2}(u, v), x_{3}(u, v)\right) \frac{\partial\left(x_{3}, x_{1}\right)}{\partial(u, v)} d u d v \\
& -\frac{1}{V(B)} \int_{a c}^{b d} \int_{3} \alpha_{3}\left(x_{3}(u, v)-y_{3}\right) f\left(x_{1}(u, v), x_{2}(u, v), x_{3}(u, v)\right) \frac{\partial\left(x_{1}, x_{2}\right)}{\partial(u, v)} d u d v \mid \\
& \quad \leq \sum_{k=1}^{3} \sum_{j=1}^{3}\left|\alpha_{k}\right|\left\|\frac{\partial^{2} f}{\partial x_{k} \partial x_{j}}\right\|_{B, \infty} \frac{1}{V(B)} \int_{B}\left|y_{k}-x_{k}\right|\left|x_{j}-y_{j}\right| d x
\end{aligned}
$$

for all $\left(y_{1}, y_{2}, y_{3}\right) \in B$ and $\alpha_{k} \in \mathbb{C}, k \in\{1,2,3\}$, with $\alpha_{1}+\alpha_{2}+\alpha_{3}=1$.

In particular, we have

$$
\begin{aligned}
& \mid \frac{1}{V(B)} \int_{B} f(x) d x-\frac{1}{3} \sum_{k=1}^{3} \frac{\partial f\left(y_{1}, y_{2}, y_{3}\right)}{\partial x_{k}}\left(y_{k}-\overline{x_{B, k}}\right) \\
& -\frac{1}{3 V(B)} \int_{a c}^{b} \int_{c}^{d}\left(x_{1}(u, v)-y_{1}\right) f\left(x_{1}(u, v), x_{2}(u, v), x_{3}(u, v)\right) \frac{\partial\left(x_{2}, x_{3}\right)}{\partial(u, v)} d u d v \\
& -\frac{1}{3 V(B)} \int_{a c}^{b} \int_{c}^{d}\left(x_{2}(u, v)-y_{2}\right) f\left(x_{1}(u, v), x_{2}(u, v), x_{3}(u, v)\right) \frac{\partial\left(x_{3}, x_{1}\right)}{\partial(u, v)} d u d v \\
& -\frac{1}{3 V(B)} \int_{a c}^{b d}\left(x_{3}(u, v)-y_{3}\right) f\left(x_{1}(u, v), x_{2}(u, v), x_{3}(u, v)\right) \frac{\partial\left(x_{1}, x_{2}\right)}{\partial(u, v)} d u d v \mid
\end{aligned}
$$




$$
\leq \frac{1}{3} \sum_{k=1}^{3} \sum_{j=1}^{3}\left\|\frac{\partial^{2} f}{\partial x_{k} \partial x_{j}}\right\|_{B, \infty} \frac{1}{V(B)} \int_{B}\left|y_{k}-x_{k}\right|\left|x_{j}-y_{j}\right| d x
$$

for all $\left(y_{1}, y_{2}, y_{3}\right) \in B$.

We also have the centre of gravity inequalities

$$
\mid \frac{1}{V(B)} \int_{B} f(x) d x
$$

$-\frac{1}{V(B)} \int_{a c}^{b} \int_{c}^{d} \alpha_{1}\left(x_{1}(u, v)-\overline{x_{B, 1}}\right) f\left(x_{1}(u, v), x_{2}(u, v), x_{3}(u, v)\right) \frac{\partial\left(x_{2}, x_{3}\right)}{\partial(u, v)} d u d v$

$-\frac{1}{V(B)} \int_{a c}^{b d} \int_{c}^{d} \alpha_{2}\left(x_{2}(u, v)-\overline{x_{B, 2}}\right) f\left(x_{1}(u, v), x_{2}(u, v), x_{3}(u, v)\right) \frac{\partial\left(x_{3}, x_{1}\right)}{\partial(u, v)} d u d v$

$-\frac{1}{V(B)} \int_{a c}^{b} \int_{c}^{d} \alpha_{3}\left(x_{3}(u, v)-\overline{x_{B, 3}}\right) f\left(x_{1}(u, v), x_{2}(u, v), x_{3}(u, v)\right) \frac{\partial\left(x_{1}, x_{2}\right)}{\partial(u, v)} d u d v \mid$

$$
\leq \sum_{k=1}^{3} \sum_{j=1}^{3}\left|\alpha_{k}\right|\left\|\frac{\partial^{2} f}{\partial x_{k} \partial x_{j}}\right\|_{B, \infty} \frac{1}{V(B)} \int_{B}\left|\overline{x_{B, k}}-x_{k}\right|\left|x_{j}-\overline{x_{B, j}}\right| d x
$$

for all $\alpha_{k} \in \mathbb{C}, k \in\{1,2,3\}$, with $\alpha_{1}+\alpha_{2}+\alpha_{3}=1$.

In particular,

$$
\mid \frac{1}{V(B)} \int_{B} f(x) d x
$$

$-\frac{1}{3 V(B)} \int_{a c}^{b d}\left(x_{1}(u, v)-\overline{x_{B, 1}}\right) f\left(x_{1}(u, v), x_{2}(u, v), x_{3}(u, v)\right) \frac{\partial\left(x_{2}, x_{3}\right)}{\partial(u, v)} d u d v$

$$
-\frac{1}{3 V(B)} \int_{a c}^{b d}\left(x_{2}(u, v)-\overline{x_{B, 2}}\right) f\left(x_{1}(u, v), x_{2}(u, v), x_{3}(u, v)\right) \frac{\partial\left(x_{3}, x_{1}\right)}{\partial(u, v)} d u d v
$$

$$
\begin{aligned}
&-\frac{1}{3 V(B)} \int_{a c}^{b d}\left(x_{3}(u, v)-\overline{x_{B, 3}}\right) f\left(x_{1}(u, v), x_{2}(u, v), x_{3}(u, v)\right) \frac{\partial\left(x_{1}, x_{2}\right)}{\partial(u, v)} d u d v \mid \\
& \leq \frac{1}{3} \frac{1}{V(B)}\left[\left\|\frac{\partial^{2} f}{\partial x_{1}^{2}}\right\|_{B, \infty} \int_{B}\left(\overline{x_{B, 1}}-x_{1}\right)^{2} d x\right. \\
&\left.+\left\|\frac{\partial^{2} f}{\partial x_{2}^{2}}\right\|_{B, \infty} \int_{B}\left(\overline{x_{B, 2}}-x_{2}\right)^{2} d x+\left\|\frac{\partial^{2} f}{\partial x_{3}^{2}}\right\|_{B, \infty} \int_{B}\left(\overline{x_{B, 3}}-x_{3}\right)^{2} d x\right] \\
&+\frac{2}{3} \frac{1}{V(B)}\left[\left\|\frac{\partial^{2} f}{\partial x_{1} \partial x_{2}}\right\|_{B, \infty}\left|\int_{B}\right| \overline{x_{B, 1}}-x_{1}|| x_{2}-\overline{x_{B, 2}} \mid d x\right.
\end{aligned}
$$




$$
\begin{aligned}
& +\left\|\frac{\partial^{2} f}{\partial x_{2} \partial x_{3}}\right\|_{B, \infty} \int_{B}\left|\overline{x_{B, 2}}-x_{2}\right|\left|x_{3}-\overline{x_{B, 3}}\right| d x \\
& \left.+\left\|\frac{\partial^{2} f}{\partial x_{1} \partial x_{3}}\right\|_{B, \infty} \int_{B}\left|\overline{x_{B, 1}}-x_{1}\right|\left|x_{3}-\overline{x_{B, 3}}\right| d x\right] .
\end{aligned}
$$

7. Example for 3-dimensional balls. Consider the 3-dimensional ball centred at $C=(a, b, c)$ and having radius $R>0$,

$$
B(C, R):=\left\{(x, y, z) \in \mathbb{R}^{3} \mid(x-a)^{2}+(y-b)^{2}+(z-c)^{2} \leq R^{2}\right\}
$$

and the sphere

$$
S(C, R):=\left\{(x, y, z) \in \mathbb{R}^{3} \mid(x-a)^{2}+(y-b)^{2}+(z-c)^{2}=R^{2}\right\} .
$$

Consider the parametrisation of $B(C, R)$ and $S(C, R)$ given by $B(C, R):\left\{\begin{array}{l}x=r \cos \psi \cos \varphi+a, \\ y=r \cos \psi \sin \varphi+b, \\ z=r \sin \psi+c,\end{array} \quad(r, \psi, \varphi) \in[0, R] \times[-\pi / 2, \pi / 2] \times[0,2 \pi]\right.$, and

$$
S(C, R):\left\{\begin{array}{l}
x=R \cos \psi \cos \varphi+a, \\
y=R \cos \psi \sin \varphi+b, \\
z=R \sin \psi+c
\end{array} \quad(\psi, \varphi) \in[-\pi / 2, \pi / 2] \times[0,2 \pi] .\right.
$$

Observe that

$$
\begin{aligned}
& \left|\begin{array}{ll}
\frac{\partial y}{\partial \psi} & \frac{\partial z}{\partial \psi} \\
\frac{\partial y}{\partial \varphi} & \frac{\partial z}{\partial \varphi}
\end{array}\right|=-R^{2} \cos ^{2} \psi \cos \varphi \\
& \left|\begin{array}{ll}
\frac{\partial x}{\partial \psi} & \frac{\partial z}{\partial \psi} \\
\frac{\partial x}{\partial \varphi} & \frac{\partial z}{\partial \varphi}
\end{array}\right|=R^{2} \cos ^{2} \psi \sin \varphi \\
& \left|\begin{array}{ll}
\frac{\partial x}{\partial \psi} & \frac{\partial y}{\partial \psi} \\
\frac{\partial x}{\partial \varphi} & \frac{\partial y}{\partial \varphi}
\end{array}\right|=-R^{2} \sin \psi \cos \psi
\end{aligned}
$$

Let us consider the transformation $T_{2}: \mathbb{R}^{3} \rightarrow \mathbb{R}^{3}$ given by

$$
T_{2}(r, \psi, \varphi):=(r \cos \psi \cos \varphi+a, r \cos \psi \sin \varphi+b, r \sin \psi+c) .
$$

It is well known that the Jacobian of $T_{2}$ is

$$
J\left(T_{2}\right)=r^{2} \cos \psi
$$

and $T_{2}$ is a one-to-one mapping defined on $[0, R] \times[-\pi / 2, \pi / 2] \times[0,2 \pi]$, with values in $B(C, R)$. Thus we have the change of variables 


$$
\iiint_{B(C, R)} f(x, y, z) d x d y d z
$$

$\begin{array}{lll}R \pi / 2 & 2 \pi\end{array}$

$=\int_{0} \int_{-\pi / 2} \int_{0} f(r \cos \psi \cos \varphi+a, r \cos \psi \sin \varphi+b, r \sin \psi+c) r^{2} \cos \psi d r d \psi d \varphi$.

We also have

$$
\begin{aligned}
\iiint_{B(C, R)}\left|z-\overline{z_{B(C, R)}}\right|^{2} d x d y d z & =\int_{0}^{R} \int_{-\pi / 2}^{\pi / 2} \int_{0}^{2 \pi} r^{2} \sin ^{2} \psi r^{2} \cos \psi d r d \psi d \varphi \\
& =\int_{0}^{R} \int_{-\pi / 2}^{\pi / 2} \int_{0}^{2 \pi} r^{4} \sin ^{2} \psi \cos \psi d r d \psi d \varphi=\frac{4}{15} \pi R^{5},
\end{aligned}
$$

and similarly

$$
\iiint_{B(C, R)}\left|x-\overline{x_{B(C, R)}}\right|^{2} d x d y d z=\iiint_{B(C, R)}\left|y-\overline{y_{B(C, R)}}\right|^{2} d x d y d z=\frac{4}{15} \pi R^{5} .
$$

Also

$$
\begin{aligned}
\iiint_{B(C, R)}\left|x-\overline{x_{B(C, R)}}\right|\left|y-\overline{y_{B(C, R)}}\right| d x d y d z & \\
= & \int_{0}^{R} \int_{-\pi / 2}^{\pi / 2} \int_{0}^{2 \pi}|r \cos \psi \cos \varphi||r \cos \psi \sin \varphi| r^{2} \cos \psi d r d \psi d \varphi \\
= & \int_{0}^{R} \int_{-\pi / 2}^{\pi / 2} \int_{0}^{2 \pi} r^{4} \cos ^{3} \psi|\sin \varphi \cos \varphi| d r d \psi d \varphi=\frac{8}{15} R^{5},
\end{aligned}
$$

and similarly

$$
\begin{aligned}
& \iiint_{B(C, R)}\left|x-\overline{x_{B(C, R)}}\right|\left|z-\overline{z_{B(C, R)}}\right| d x d y d z \\
& \quad=\iiint_{B(C, R)}\left|y-\overline{y_{B(C, R)}}\right|\left|z-\overline{z_{B(C, R)}}\right| d x d y d z=\frac{8}{15} R^{5} .
\end{aligned}
$$

Since $V(B(C, R))=\frac{4 \pi R^{3}}{3}$, by 6.4 we get

$$
\begin{aligned}
\frac{1}{\frac{4 \pi R^{3}}{3}} \int_{0}^{R} \int_{-\pi / 2}^{\pi / 2} \int_{0}^{2 \pi} f(r \cos \psi \cos \varphi+a, r \cos \psi \sin \varphi & +b, r \sin \psi+c) \\
& \times r^{2} \cos \psi d r d \psi d \varphi
\end{aligned}
$$


$+\frac{1}{4 \pi} \int_{-\pi / 2}^{\pi / 2} \int_{0}^{2 \pi} f(R \cos \psi \cos \varphi+a, R \cos \psi \sin \varphi+b, R \sin \psi+c) \cos ^{3} \psi \cos ^{2} \varphi d \psi d \varphi$ $-\frac{1}{4 \pi} \int_{-\pi / 2}^{\pi / 2} \int_{0}^{2 \pi} f(R \cos \psi \cos \varphi+a, R \cos \psi \sin \varphi+b, R \sin \psi+c) \cos ^{3} \psi \sin ^{2} \varphi d \psi d \varphi$ $+\frac{1}{4 \pi} \int_{-\pi / 2}^{\pi / 2} \int_{0}^{2 \pi} f(R \cos \psi \cos \varphi+a, R \cos \psi \sin \varphi+b, R \sin \psi+c) \sin ^{2} \psi \cos \psi d \psi d \varphi$ $\leq \frac{1}{15} R^{2}\left[\left\|\frac{\partial^{2} f}{\partial x^{2}}\right\|_{B(C, R), \infty}+\left\|\frac{\partial^{2} f}{\partial y^{2}}\right\|_{B(C, R), \infty}+\left\|\frac{\partial^{2} f}{\partial z^{2}}\right\|_{B(C, R), \infty}\right]$ $+\frac{4}{15 \pi} R^{2}\left[\left\|\frac{\partial^{2} f}{\partial x \partial y}\right\|_{B(C, R), \infty}+\left\|\frac{\partial^{2} f}{\partial y \partial z}\right\|_{B(C, R), \infty}+\left\|\frac{\partial^{2} f}{\partial z \partial x}\right\|_{B(C, R), \infty}\right]$.

Acknowledgements. The author would like to thank the anonymous referee for valuable comments that have been implemented in the final version of the paper.

\section{References}

[1] T. M. Apostol, Calculus. Volume II, Multi-Variable Calculus and Linear Algebra, with Applications to Differential Equations and Probability, 2nd ed., Wiley, New York, 1969.

[2] N. S. Barnett, F. C. Cîrstea and S. S. Dragomir, Some inequalities for the integral mean of Hölder continuous functions defined on disks in a plane, in: Inequality Theory and Applications, Vol. 2 (Chinju/Masan, 2001), Nova Sci. Publ., Hauppauge, NY, 2003, 7-18; https://rgmia.org/papers/v5n1/BCD.pdf

[3] N. S. Barnett and S. S. Dragomir, An Ostrowski type inequality for double integrals and applications for cubature formulae, Soochow J. Math. 27 (2001), 1-10.

[4] N. S. Barnett, S. S. Dragomir and C. E. M. Pearce, A quasi-trapezoid inequality for double integrals, ANZIAM J. 44 (2003), 355-364.

[5] H. Budak and M. Z. Sarıkaya, An inequality of Ostrowski-Grüss type for double integrals, Stud. Univ. Babeş-Bolyai Math. 62 (2017), 163-173.

[6] S. S. Dragomir, P. Cerone, N. S. Barnett and J. Roumeliotis, An inequality of the Ostrowski type for double integrals and applications for cubature formulae, Tamsui Oxford J. Math. Sci. 16 (2000), 1-16.

[7] S. S. Dragomir, New inequalities for double and path integrals on general domains via Green's identity, preprint RGMIA Res. Rep. Coll. 22 (2019), art. 57, 18 pp.; https://rgmia.org/papers/v22/v22a57.pdf.

[8] S. Erden and M. Z. Sarikaya, On exponential Pompeiu's type inequalities for double integrals with applications to Ostrowski's inequality, New Trends Math. Sci. 4 (2016), 256-267.

[9] G. Hanna, Some results for double integrals based on an Ostrowski type inequality, in: Ostrowski Type Inequalities and Applications in Numerical Integration, Kluwer, Dordrecht, 2002, 331-371. 
[10] G. Hanna, S. S. Dragomir and P. Cerone, A general Ostrowski type inequality for double integrals, Tamkang J. Math. 33 (2002), 319-333.

[11] Z. Liu, A sharp general Ostrowski type inequality for double integrals, Tamsui Oxford J. Information Math. Sci. 28 (2012), 217-226.

[12] M. E. Özdemir, A. O. Akdemir and E. Set, A new Ostrowski-type inequality for double integrals, J. Inequal. Spec. Funct. 2 (2011), 27-34.

[13] B. G. Pachpatte, A new Ostrowski type inequality for double integrals, Soochow J. Math. 32 (2006), 317-322.

[14] M. Z. Sarikaya, On the Ostrowski type integral inequality for double integrals, Demonstratio Math. 45 (2012), 533-540.

[15] M. Z. Sarikaya and H. Ogunmez, On the weighted Ostrowski-type integral inequality for double integrals, Arab. J. Sci. Engrg. 36 (2011), 1153-1160.

[16] M. Singer, The divergence theorem, https://www.maths.ed.ac.uk/ jmf/Teaching/ Lectures/divthm.pdf

Silvestru Sever Dragomir

Mathematics, College of Engineering \& Science

Victoria University

PO Box 14428

Melbourne, MC 8001, Australia

ORCID: 0000-0003-2902-6805

E-mail: sever.dragomir@vu.edu.au

http://rgmia.org/dragomir

and

DST-NRF Centre of Excellence in the Mathematical and Statistical Sciences

School of Computer Science \& Applied Mathematics

University of the Witwatersrand

Private Bag 3

Johannesburg 2050, South Africa 